\title{
An unusual presentation of pancreatic neuroendocrine tumour (PNET)
}

\author{
Authors: Anup Halappanavar ${ }^{A}$ and Rajeev Pakhetra ${ }^{B}$
}

Insulinoma is a rare pancreatic endocrine tumour derived from $\beta$ cells that secrete insulin, which results in recurrent hypoglycaemia. Most are benign and solitary. The age range for peak incidence is between 30 and 60 years (median 47 years) and it is more prevalent in women. Diagnosis relies on clinical features along with laboratory tests and imaging. However, non-specific symptoms and the small size of the tumour can lead to misdiagnosis and difficult localisation. Clinical presentation is due to hypoglycaemia secondary to excessive and uncontrolled secretion of insulin and involves neuroglycopaenic (neuronal glucose deprivation, can cause death) and neurogenic (autonomic nervous system discharge) symptoms.

\section{Case report}

A 64-year-old woman was admitted to the emergency department (ED) with history of abnormal behaviour (facial grimacing, bouts of crying/shouting, bizarre uncoordinated limb movements) over the past 6 years. She was previously misdiagnosed as dissociative disorder and treated with antipsychotics and electroconvulsive therapy (ECT).

During initial evaluation at ED, random blood glucose was documented to be $27 \mathrm{mg} / \mathrm{dL}$ which reversed with intravenous (IV) $25 \%$ dextrose bolus. Screening for sulfonylureas was negative. Further evaluation revealed increased levels of fasting serum insulin $28.60 \mu \mathrm{U} / \mathrm{mL}$ (normal, 2.0-25.0) and serum C-peptide $6.08 \mathrm{ng} / \mathrm{mL}$ (normal, 0.81-3.85). Triple-phase computed tomography (CT) showed a $15 \mathrm{~mm}$ discrete lesion projecting superiorly from the proximal body of the pancreas (Fig 1). However, a ${ }^{68} \mathrm{Ga}$ DOTATATE scan revealed no focal lesion with octreotide receptor expression within the pancreas. She was managed with IV $10 \%$ dextrose infusion and taken up for surgical excision of the exophytic pancreatic mass. Following surgery, the patient has been euglycaemic and is off dextrose infusion presently, with no neuroglycopaenic or autonomic symptoms.

\section{Conclusion}

Insulinoma remains a diagnostic challenge since symptoms are non-specific and may lead to incorrect diagnosis. As in this case, a psychiatric illness might be wrongly considered as

Authors: ${ }^{\mathrm{A}}$ Armed Forces Medical College, Pune, India; ${ }^{\mathrm{B}}$ Command Hospital (Southern Command), Pune, India
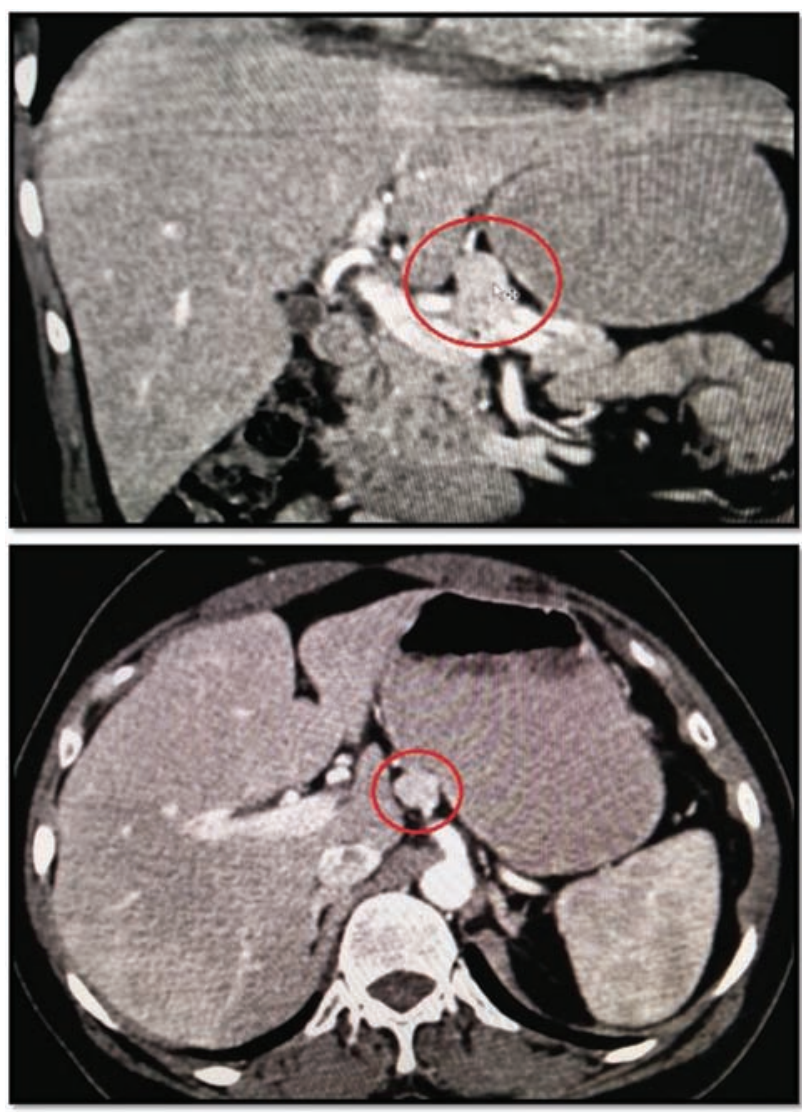

Fig 1. Computed tomography shows discrete lesion projecting superiorly from the proximal body of the pancreas

the culprit. Neuropsychiatric symptoms are a common clinical presentation of an insulinoma. A high level of clinical expertise is crucial to avoid misdiagnoses with psychiatric illnesses before insulinoma is recognised. It can be easily confirmed if it fulfils Whipple's triad, standard endocrine tests and is curable by surgery. On the other hand, severe and sustained hypoglycaemia due to misdiagnosis can lead to disability/death.

\section{Conflict of interest statement}

None declared. 


\section{References}

1 Tarchouli M, Ali AA, Ratbi MB et al. Long-standing insulinoma: two case reports and review of the literature. BMC Res Notes 2015;8:444.

2 Renca S, Cerejeira J, Santos G. P-243 - An insulinoma presenting with neuropsychiatric symptoms. Eur Psychiatry 2012;27:1.

3 Okabayashi T, Shima Y, Sumiyoshi T et al. Diagnosis and management of insulinoma. World J Gastroenterol 2013;19:829-37.
4 Placzkowski KA, Vella A, Thompson GB et al. Secular trends in the presentation and management of functioning insulinoma at the Mayo Clinic, 1987-2007. J Clin Endocrinol Metab 2009;94:106973.

5 Rassouli A, Lai JH, Sargeant R. Insulinoma - an atypical presentation: case report and literature review. Univ Toronto Med J 2004:82:36-41. 